\title{
Heterozygous connexin 50 mutation affects metabolic syndrome attributes in spontaneously hypertensive rat
}

Ondřej Šeda ${ }^{1,2^{*}}$, Drahomíra Křenová, Olena Oliyarnyk ${ }^{3}$, Lucie Šedová1,2, Michaela Krupková ${ }^{1}$, František Liška', Blanka Chylíková', Ludmila Kazdová ${ }^{3}$ and Vladimír Křen ${ }^{1}$

\begin{abstract}
Background: Several members of connexin family of transmembrane proteins were previously implicated in distinct metabolic conditions. In this study we aimed to determine the effects of complete and heterozygous form of connexin50 gene (Gja8) mutation L7Q on metabolic profile and oxidative stress parameters in spontaneously hypertensive inbred rat strain (SHR).

Methods: Adult, standard chow-fed male rats of SHR, heterozygous SHR-Dca+/- and SHR-Dca-/- coisogenic strains were used. At the age of 4 months, dexamethasone $(2.6 \mu \mathrm{g} / \mathrm{ml})$ was administered in the drinking water for three days. The lipidemic profile (cholesterol and triacylglycerol concentration in 20 lipoprotein fractions, chylomicron, VLDL, LDL and HDL particle sizes) together with 33 cytokines and hormones in serum and several oxidative stress parameters in plasma, liver, kidney and heart were assessed.

Results: SHR and SHR-Dca-/- rats had similar concentrations of triacylglycerols and cholesterol in all major lipoprotein fractions. The heterozygotes reached significantly highest levels of total (SHR-Dca+/-: $51.3 \pm 7.2 \mathrm{vs.}$ SHR: $34.5 \pm 2.4$ and SHR-Dca-/-: $34.4 \pm 2.5 \mathrm{mg} / \mathrm{dl}, p=0.026$ ), chylomicron and VLDL triacylglycerols. The heterozygotes showed significantly lowest values of HDL cholesterol $(40.9 \pm 2.3 \mathrm{mg} / \mathrm{dl})$ compared both to SHR $(51.8 \pm 2.2 \mathrm{mg} / \mathrm{dl})$ and SHR-Dca-/- $(48.6 \pm 2.7 \mathrm{mg} / \mathrm{dl})$. Total and LDL cholesterol in SHR-Dca+/- was lower compared to SHR. Glucose tolerance was improved and insulin concentrations were lowest in SHR-Dca-/- (1. $11 \pm 0.20 \mathrm{pg} / \mathrm{ml})$ in comparison with both SHR $(2.32 \pm 0.49 \mathrm{pg} / \mathrm{ml})$ and SHR-Dca+/- $(3.04 \pm 0.21 \mathrm{pg} / \mathrm{ml})$. The heterozygous rats showed profile suggestive of increased oxidative stress as well as highest serum concentrations of several pro-inflammatory cytokines including interleukins $6,12,17,18$ and tumor necrosis factor alpha.
\end{abstract}

Conclusions: Our results demonstrate that connexin50 mutation in heterozygous state affects significantly the lipid profile and the oxidative stress parameters in the spontaneously hypertensive rat strain.

Keywords: Connexin, Animal models, Lipoprotein, Oxidative stress, Metabolic syndrome

\footnotetext{
* Correspondence: ondrej.seda@lf1.cuni.cz

${ }^{1}$ Institute of Biology and Medical Genetics, First Faculty of Medicine, Charles

University, Albertov 4, 12800 Prague 2, Czech Republic

${ }^{2}$ Division BIOCEV, Institute of Molecular Genetics of the Academy of Sciences

of the Czech Republic, Laboratory of Rat Models of Metabolic Disorders,

Vídeňská 1083, 14220 Prague 4, Czech Republic

Full list of author information is available at the end of the article
}

(c) The Author(s). 2016 Open Access This article is distributed under the terms of the Creative Commons Attribution 4.0 International License (http://creativecommons.org/licenses/by/4.0/), which permits unrestricted use, distribution, and reproduction in any medium, provided you give appropriate credit to the original author(s) and the source, provide a link to the Creative Commons license, and indicate if changes were made. The Creative Commons Public Domain Dedication waiver (http://creativecommons.org/publicdomain/zero/1.0/) applies to the data made available in this article, unless otherwise stated. 


\section{Background}

The rapidly increasing incidence and prevalence of dyslipidemia, obesity and type 2 diabetes is becoming a major global health issue, however, the need for identification of relevant molecular targets and devising effective preventive and therapeutic algorithms is met by only modest advancement of our knowledge. Historically, several major definitions of metabolic syndrome have been used, yet in 2009, five major scientific organizations released a joint interim statement leading to a unified definition of the disorder [1]. Individuals who meet at least 3 out of 5 criteria [elevated waist circumference, elevated triglycerides, reduced high-density lipoprotein cholesterol, elevated blood pressure and elevated fasting glucose (or treatment of these conditions)] are diagnosed with metabolic syndrome. Exact thresholds are defined for all criteria, except elevated waist circumference, which must rely on population and country-specific definitions [1]. All individual features of metabolic syndrome are complex traits with relatively balanced strengths of heritable and environmental components (the heritability of individual components ranges from 40-70\%) [2]. The detailed analysis of genetic component of complex traits in general human population encounters numerous hurdles, including reduced penetrance, expressivity, ethnical admixture and other population stratifications, epigenetic and environmental influences [3]. Also, it is not only the fully manifested condition that is of clinical relevance; evidence is mounting with regard to the importance of early stages of metabolic syndrome components, particularly impaired glucose tolerance and prediabetes [4]. In this context, experimental models can greatly facilitate the process of deconstruction of genetic architecture of multifactorial traits [5]. In rodents and humans, a family of highly conserved transmembrane proteins forming intercellular gap junction channels called connexins comprises 21 isoforms [6]. The tissue distribution pattern of many connexins and their close functional connection to multiple metabolic and signaling pathways that are crucial for insulin sensitivity, lipid homeostasis and hemodynamic regulation makes them plausible candidates for metabolic syndrome pathogenesis [7]. We have previously established that a spontaneous mutation L7Q in Gja8 gene (coding for connexin50) which arose in spontaneously hypertensive rat strain (SHR hereafter; Rat Genome Database (RGD) [8] ID: 631848) leads to microphthalmia and cataract [9]. The resulting mutant coisogenic strain SHR-Gja $8^{\mathrm{mlCub}}$ (SHR-Dca-/- hereafter, RGD ID: 2293729) shows decreased blood pressure compared to SHR [10]. This study aimed to further explore the effects of connexin 50 mutation on metabolic and cytokine profile in SHR-Dca-/and SHR-Dca+/- strains including a battery of parameters of oxidative stress in the animals challenged by dexamethasone, a dyslipidemia and insulin resistanceinducing glucocorticoid $[11,12]$.

\section{Methods \\ Experimental protocol}

Adult male rats were housed under temperature- and humidity-controlled conditions with a $12 \mathrm{~h} / 12 \mathrm{~h}$ lightdark cycle. Animals had free access to food (standard chow) and water at all times. At 4 months of age, males from the SHR, SHR-Dca+/- and SHR-Dca-/- strains $(n=8 /$ strain/procedure) were administered dexamethasone (Dexamed, Medochemie) in drinking water (2.6 $\mu \mathrm{g} / \mathrm{ml}$ ) for three days as described previously $[11,12]$. Subsequently, they were subjected to an oral glucose tolerance test (OGTT) after overnight fasting and blood samples were drawn. The animals were then sacrificed, and their total weight and the weights of the heart, liver, kidneys, and adrenal glands, and the epididymal and retroperitoneal fat pads, were determined.

\section{Metabolic measurements}

The OGTT was performed after overnight fasting. Blood samples for glycemic assessment (Ascensia Elite Blood Glucose Meter, Bayer HealthCare, Mishawaka, IN, USA; validated by the Institute of Clinical Biochemistry and Laboratory Diagnostics of the First Faculty of Medicine, Charles University in Prague) were obtained from the tail vein at intervals of $0,30,60,120$, and 180 min after intragastric glucose administration to conscious rats $(3 \mathrm{~g} / \mathrm{kg}$ body weight, $30 \%$ aqueous solution). The lipid profile (cholesterol and triacylglycerols blood concentration in 20 lipoprotein fractions, glycerol level and chylomicron, VLDL, LDL and HDL particle sizes) was assessed by high performance liquid chromatography method as described previously [13, 14]. Enzyme-linked immunosorbent assay (ELISA) kits were used to determine the serum levels of adiponectin (BioSource, San Diego, CA, USA). Milliplex Rat Metabolic Hormone Magnetic Bead panel (Merck Millipore, Darmstadt, Germany) was used for the simultaneous quantification of C-peptide, gastric inhibitory polypeptide (GIP), glucagon-like polypeptide-1 (GLP1), pancreatic polypeptide (PP), protein tyrosine tyrosine (PYY), glucagon, insulin and leptin, the Bio-Plex Pro Rat Cytokine 24-Plex Immunoassay (Bio-Rad, Hercules, CA, USA) was used to assess the concentrations of erythropoietin (EPO), Granulocyte-colony stimulating factor (GCSF), Granulocyte-macrophage colony-stimulating factor (GM-CSF), chemokine (C-X-C motif) ligand 1 (GRO/KC), interferon gamma (IFN- $\gamma$ ), interleukins IL- $1 \alpha$, IL-1 $\beta$, IL-2, IL-4, IL-5, IL-6, IL-7, IL-10, IL-12p70, IL-13, IL-17A, IL18, macrophage colony-stimulating factor (M-CSF), monocyte chemotactic protein 1 (MCP-1), macrophage inflammatory protein 1-alpha (MIP-1 $\alpha)$, macrophage

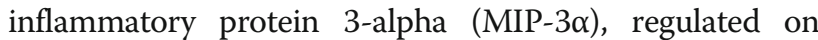


activation, normal $\mathrm{T}$ cell expressed and secreted (RANTES), tumor necrosis factor alpha (TNF- $\alpha$ ) and vascular endothelial growth factor (VEGF), using BioPlex system (Bio-Rad, Hercules, CA, USA).

The oxidation stress parameters were assessed as described previously $[15,16]$. In short, the activity of superoxide dismutase (SOD) was determined spectrophotometrically using p-tetrazolin nitro-blue, the activity of catalase (CAT) was measured spectrophotometrically by ammonium molybdate reaction with $\mathrm{H}_{2} \mathrm{O}_{2}$, and the activity of glutathione peroxidase (GSH-Px) and content of glutathione (GSH) were analyzed spectrophotometrically using Ellman's reagent. Glutathione reductase activity was measured using commercially available assay kit (Sigma-Aldrich, St. Louis, MO, USA). The level of conjugated dienes $(\mathrm{CD})$ was determined spectrophotometrically after extraction into n-heptane. Thiobarbituric acid reactive substances (TBARS) were measured according to Naito et al. [17].

\section{Statistical analysis}

All statistical analyses were performed using STATISTICA $12 \mathrm{CZ}$. The metabolic and morphometric data were compared by one-way analyses of variance (ANOVA) with STRAIN as main factor followed by Tukey's honest significance difference test for detailed pair-wise comparison. The null hypothesis was rejected whenever $p<0.05$.

\section{Results}

\section{Morphometric and metabolic profile}

The body weights and the relative weights of major organs were comparable among the three genotypes. The relative weight of the adipose tissue depots was decreased in the SHR-Dca-/- rats and increased in the heterozygotes compared to SHR, in both cases reaching statistical significance for the epididymal fat pad (Table 1). Glucose tolerance was improved in SHR-Dca-/- in comparison to both SHR and SHR-Dca+/- strains as reflected by significantly lowest values of glycaemia throughout the oral glucose tolerance test (Fig. 1) combined with lower insulin and C-peptide concentrations (Table 1). The heterozygous rats showed the highest concentrations of gastric inhibitory polypeptide of the three tested strains. Glucagon, glucagon-like polypeptide-1, pancreatic polypeptide, protein tyrosine tyrosine, adiponectin and leptin levels did not differ among the three strains (Table 1).

\section{Detailed lipid profile}

While SHR and SHR-Dca-/- rats had similar concentrations of triacylglycerols and cholesterol in all major lipoprotein fractions, the heterozygotes reached significantly highest levels of total, chylomicron (CM) and very lowdensity lipoprotein (VLDL) triacylglycerols of the three tested strains (Table 2). As shown in Fig. 2, this difference was evident throughout most CM and VLDL subfractions, further corroborated by significantly largest VLDL particles

Table 1 Morphometric and metabolic profile of SHR, SHR-Dca +/- and SHR-Dca -/- male rats

\begin{tabular}{|c|c|c|c|c|}
\hline Trait & SHR & SHR-Dca+/- & SHR-Dca-I- & $p$-value \\
\hline Body weight (b.wt.), g & $299 \pm 6$ & $299 \pm 9$ & $281 \pm 8$ & 0.16 \\
\hline Liver, g/100 g b.wt. & $3.19 \pm 0.02$ & $3.29 \pm 0.08$ & $3.16 \pm 0.09$ & 0.41 \\
\hline Heart, g/100 g b.wt. & $0.44 \pm 0.01$ & $0.45 \pm 0.01$ & $0.45 \pm 0.01$ & 0.99 \\
\hline Kidney, g/100 g b.wt. & $0.77 \pm 0.01$ & $0.77 \pm 0.01$ & $0.77 \pm 0.01$ & 0.98 \\
\hline Adrenals, mg/100 g b.wt. & $8.46 \pm 0.04$ & $8.50 \pm 0.04$ & $9.58 \pm 0.07$ & 0.21 \\
\hline EFP wt., g/100 g b.wt. & $1.05 \pm 0.03$ & $1.14 \pm 0.03^{b}$ & $0.92 \pm 0.05^{*}$ & 0.005 \\
\hline RFP wt., g/100 g b.wt. & $0.91 \pm 0.05$ & $0.98 \pm 0.05$ & $0.79 \pm 0.09$ & 0.16 \\
\hline Glycerol (mg/dl) & $2.97 \pm 0.32$ & $2.73 \pm 0.23$ & $3.12 \pm 0.30$ & 0.67 \\
\hline Adiponectin $(\mu \mathrm{g} / \mathrm{ml})$ & $5.14 \pm 0.56$ & $5.58 \pm 0.30$ & $5.42 \pm 0.24$ & 0.72 \\
\hline C-peptide, pg/ml & $615 \pm 57$ & $653 \pm 39^{a}$ & $425 \pm 42^{*}$ & 0.046 \\
\hline $\mathrm{GIP}, \mathrm{pg} / \mathrm{ml}$ & $163 \pm 25$ & $247 \pm 33^{*, a}$ & $122 \pm 20$ & 0.031 \\
\hline GLP1, pg/ml & $102 \pm 56$ & $68 \pm 41$ & $71 \pm 44$ & 0.86 \\
\hline Glucagon, pg/ml & $155 \pm 50$ & $365 \pm 111$ & $153 \pm 35$ & 0.10 \\
\hline Insulin, ng/ml & $2.32 \pm 0.49$ & $3.04 \pm 0.21^{b}$ & $1.11 \pm 0.20^{*}$ & 0.025 \\
\hline $\mathrm{PP}, \mathrm{pg} / \mathrm{ml}$ & $39.3 \pm 3.9$ & $45.1 \pm 4.3$ & $39.2 \pm 3.5$ & 0.52 \\
\hline $\mathrm{PYY}, \mathrm{pg} / \mathrm{ml}$ & $79.3 \pm 10.0$ & $96.3 \pm 15.6$ & $76.6 \pm 13.5$ & 0.53 \\
\hline Leptin, ng/ml & $6.38 \pm 0.39$ & $6.51 \pm 0.35$ & $6.41 \pm 0.52$ & 0.97 \\
\hline
\end{tabular}




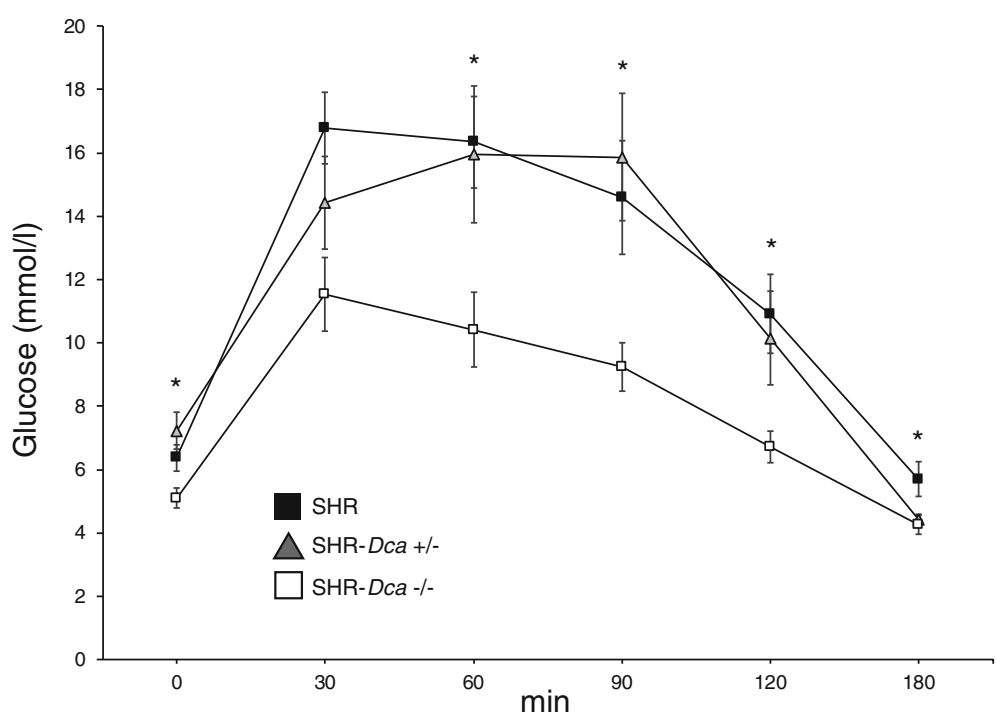

Fig. 1 Glycemic time courses for adult male SHR (black squares) vs. SHR-Dca+/- (grey triangles) and SHR-Dca-/- (white squares) adult male rats. Data are expressed as mean \pm SEM $(n=8 /$ strain). Within the graph, the significance levels of the ANOVA for STRAIN as major factor are indicated as follows: * $p<0.05$

in SHR-Dca+/- (Additional file 1: Table S1). On the other hand, total and low-density lipoprotein (LDL) cholesterol in SHR-Dca+/- was lower compared to SHR and for highdensity lipoprotein (HDL) cholesterol the heterozygotes showed significantly lowest values compared both to SHR and SHR-Dca-/- (Table 2). The detailed analysis revealed that most pronounced dissimilarities for cholesterol concentrations were found in small and very small LDL particles and in very large HDL particles (Fig. 2).

Table 2 Triacylglycerols and cholesterol concentrations in major lipoprotein subfractions in SHR, SHR-Dca+/- and SHR-Dca-/male rats

\begin{tabular}{llcll}
\hline Trait $(\mathrm{mg} / \mathrm{dl})$ & SHR & SHR-Dca+/- & SHR-Dca-/- & $p$-value \\
\hline Total TG & $34.5 \pm 2.4$ & $51.3 \pm 7.2^{* \prime} a$ & $34.4 \pm 2.5$ & 0.026 \\
Chylomicron TG & $6.20 \pm 0.87$ & $10.53 \pm 1.80^{*} a$ & $5.78 \pm 0.79$ & 0.030 \\
VLDL-TG & $15.7 \pm 1.3$ & $26.8 \pm 4.9^{*} a$ & $16.0 \pm 1.4$ & 0.025 \\
LDL-TG & $9.63 \pm 0.55$ & $9.98 \pm 0.76$ & $9.84 \pm 0.32$ & 0.91 \\
HDL-TG & $2.89 \pm 0.22$ & $3.19 \pm 0.21$ & $2.84 \pm 0.13$ & 0.42 \\
Total C & $81.4 \pm 5.2$ & $58.0 \pm 3.2+$ & $70.2 \pm 5.7$ & 0.016 \\
Chylomicron C & $0.70 \pm 0.07$ & $0.83 \pm 0.09$ & $0.63 \pm 0.06$ & 0.19 \\
VLDL-C & $2.64 \pm 0.12$ & $2.67 \pm 0.30$ & $2.19 \pm 0.15$ & 0.22 \\
LDL-C & $26.2 \pm 3.0$ & $13.6 \pm 1.3 \dagger$ & $18.7 \pm 3.0$ & 0.015 \\
HDL-C & $51.8 \pm 2.2$ & $40.9 \pm 2.3 \dagger^{\prime} a$ & $48.6 \pm 2.7$ & 0.020 \\
\hline
\end{tabular}

Triacylglycerols (TG) and cholesterol (C) concentrations in major lipoprotein subfractions (chylomicron, VLDL very low-density lipoprotein, LDL low-density lipoprotein, HDL high-density lipoprotein) in SHR, SHR-Dca+/- and SHR-Dca $-/$ - male rats. Data are shown as mean \pm SEM. The significance levels of oneway ANOVA for STRAIN as a major factor are shown in last column; significant values are italicized. ${ }^{*} p<0.05$ and $+p<0.01$ for pair-wise comparisons (posthoc Tukey's HSD test) between SHR-Dca-/- or SHR-Dca+/- strains vs. SHR. ${ }^{a} p$ $<0.05$ for pair-wise comparisons between SHR-Dca-/- and SHR-Dca+/strains. ${ }^{*} p<0.05$ vs. SHR. $+p<0.01$ vs. SHR. ${ }^{a} p<0.05$ vs. SHR-Dca-/-
Oxidative stress assessment and cytokine profile

SOD activity in liver and plasma GSH were significantly decreased both in SHR-Dca+/- and SHR-Dca-/- compared to SHR (Table 3). TBARS in plasma were significantly increased both in SHR-Dca+/- and SHR-Dca-/compared to SHR. While the SHR-Dca-/- showed comparable values to those in SHR for the rest of the assessed oxidative stress parameters, the heterozygous rats had significantly lowest activity of kidney SOD, kidney glutathione peroxidase and plasma glutathione reductase as well as significantly highest liver catalase activity compared both to SHR and SHR-Dca-/(Table 3). Also, plasma glutathione peroxidase activity was lower in SHR-Dca+/- compared to SHR. While SHR and SHR-Dca-/- did not differ across the panel of 24 cytokines, the SHR-Dca+/- showed significantly highest concentrations of interleukins IL6, IL12, IL17, IL18, granulocyte-macrophage colony-stimulating factor, macrophage inflammatory protein 3-alpha and monocyte chemotactic protein 1 (Fig. 3).

\section{Discussion}

The results of the current study demonstrate that the nonsynonymous mutation L7Q in the amino terminal domain of $\mathrm{Cx} 50$ has multiple metabolic consequences in spontaneously hypertensive rat and these vary substantially depending on the homozygous vs. heterozygous state of the mutation. The distinct phenotypic effect of heterozygous genotype has been previously observed for another member of connexin family, the GJA5 gene coding for connexin 40. While several heterozygous GJA5 mutations were identified in patients suffering from 

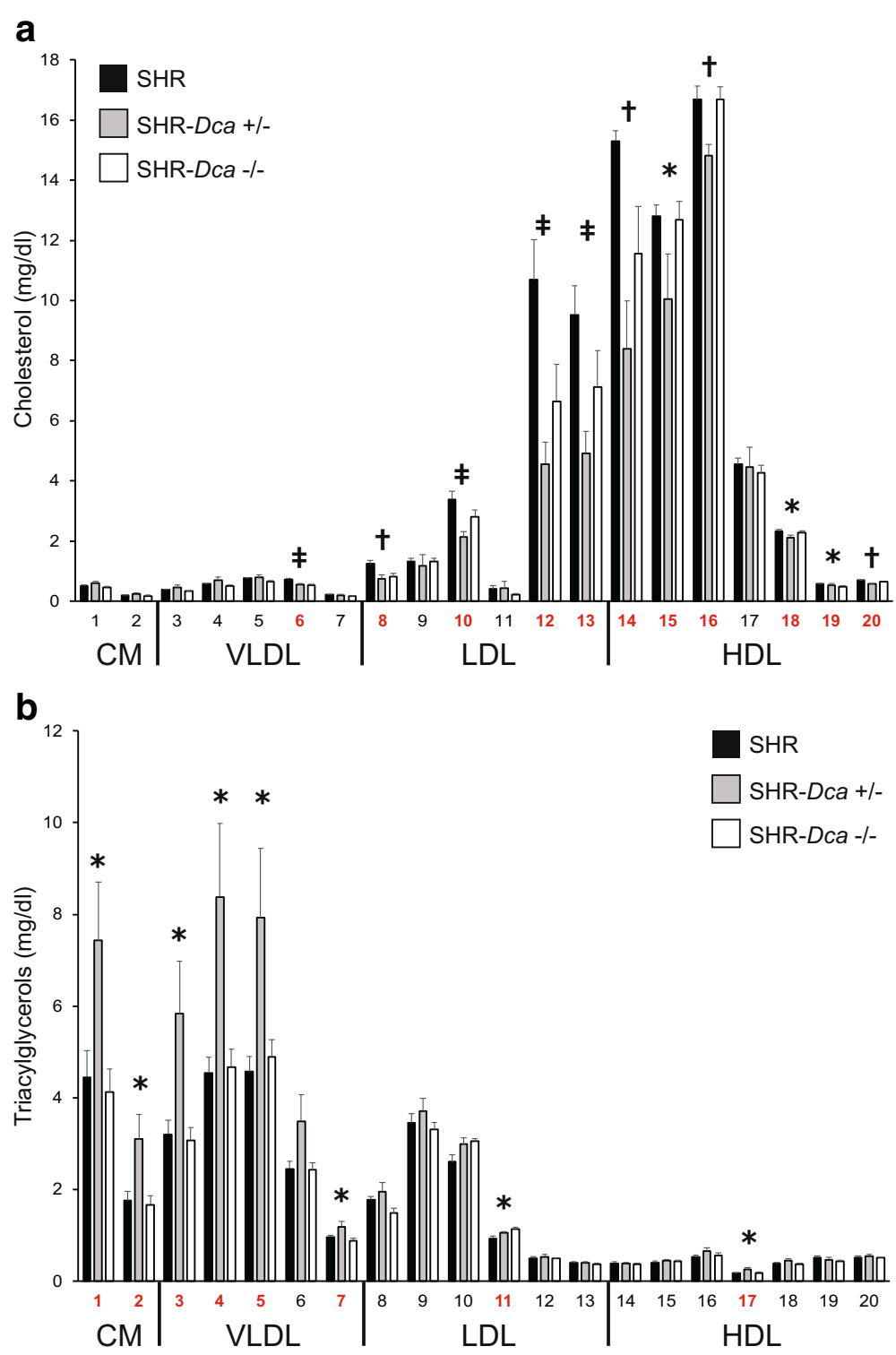

Fig. 2 The cholesterol (panel a) and triacylglycerols (panel b) content in 20 lipoprotein subfractions in SHR (black bars), SHR-Dca+/- (grey bars) and SHR-Dca-/- (open bars) adult male rats. Data are expressed as mean \pm SEM $(n=8 /$ strain). Within the graph, the significance levels of the ANOVA for STRAIN as major factor are indicated as follows: ${ }^{*} p<0.05 ; \uparrow<0.01 ; \neq p<0.001$. Labels of fractions showing significant differences between strains are highlighted in red. The allocation of individual lipoprotein subfractions to major lipoprotein classes is shown in order of particle's decreasing size from left to right. CM: chylomicron, VLDL: very low-density lipoprotein, LDL: low-density lipoprotein, HDL: high-density lipoprotein

atrial fibrillation, transgenic mice carrying one of these mutations, Cx40A96S, developed hypertension only when both alleles were mutant [18]. Further studies suggest that the gene dosage effect may be also tissuespecific [19]. It is presumed that mutations in the regions contributing to channel pore are likely to result in functional alterations [20]. In a comprehensive review of available structural and functional studies of Cx50 gap junction channels, Xin and Bai summarized compelling evidence that the amino terminal domain of Cx50 lines the pore of gap junction channels and plays an important role in single channel conductance and transjunctional voltage-dependent gating as well as in limiting the rate of ion permeation. The pore size of a gap junction channel, its switch control for opening and closing, and the modulations by chemicals can differ depending on the connexin subtypes that compose the channel. [21]. It is possible that in the heterozygous animals, heterotypic and/or heteromeric Cx50 channels are formed with both the mutated and the wild-type connexin 50 , resulting in distinctive shifts in functional properties of the connexin [22]. The ability of mutant Cx50 to 
Table 3 Oxidative stress parameters in SHR, SHR-Dca+/- and SHR-Dca-/- male rats

\begin{tabular}{|c|c|c|c|c|}
\hline Phenotype & SHR & SHR-Dca+/- & SHR-Dca-/- & $p$-value \\
\hline Plasma SOD & $0.343 \pm 0.030$ & $0.319 \pm 0.030$ & $0.283 \pm 0.034$ & 0.43 \\
\hline Liver SOD & $0.097 \pm 0.006$ & $0.074 \pm 0.005 \dagger$ & $0.082 \pm 0.003^{*}$ & 0.012 \\
\hline Kidney SOD & $0.070 \pm 0.002$ & $0.040 \pm 0.002 \neq^{b}$ & $0.059 \pm 0.008$ & 0.0010 \\
\hline Heart SOD & $0.085 \pm 0.008$ & $0.079 \pm 0.009$ & $0.083 \pm 0.009$ & 0.88 \\
\hline Plasma CAT & $341 \pm 16$ & $354 \pm 30$ & $414 \pm 55$ & 0.36 \\
\hline Liver CAT & $773 \pm 37$ & $1081 \pm 24 \neq^{, c}$ & $827 \pm 31$ & 0.000004 \\
\hline Kidney CAT & $464 \pm 19$ & $571 \pm 41$ & $539 \pm 46$ & 0.16 \\
\hline Heart CAT & $629 \pm 36$ & $679 \pm 31$ & $621 \pm 38$ & 0.44 \\
\hline Plasma GSH-Px & $546 \pm 30$ & $379 \pm 27+$ & $426 \pm 60$ & 0.027 \\
\hline Liver GSH-Px & $417 \pm 27$ & $320 \pm 18^{*}$ & $389 \pm 33$ & 0.042 \\
\hline Kidney GSH-Px & $506 \pm 31$ & $378 \pm 23+^{a}$ & $460 \pm 24$ & 0.0092 \\
\hline Heart GSH-Px & $624 \pm 35$ & $577 \pm 20$ & $612 \pm 16$ & 0.38 \\
\hline Plasma GR & $212 \pm 21$ & $158 \pm 13^{*, c}$ & $259 \pm 13$ & 0.001 \\
\hline Liver GR & $387 \pm 22$ & $430 \pm 14$ & $372 \pm 32$ & 0.20 \\
\hline Kidney GR & $273 \pm 16$ & $241 \pm 9$ & $198 \pm 31$ & 0.06 \\
\hline Heart GR & $244 \pm 24$ & $228 \pm 16$ & $192 \pm 26$ & 0.27 \\
\hline Plasma GSH & $31.0 \pm 2.0$ & $22.3 \pm 1.1 \neq$ & $21.9 \pm 1.0 \neq$ & 0.0004 \\
\hline Liver GSH & $24.6 \pm 1.5$ & $23.4 \pm 1.3$ & $28.2 \pm 1.8$ & 0.10 \\
\hline Kidney GSH & $25.1 \pm 1.7$ & $19.8 \pm 1.2$ & $24.6 \pm 1.9$ & 0.06 \\
\hline Heart GSH & $25.8 \pm 2.4$ & $27.4 \pm 2.0$ & $25.5 \pm 1.2$ & 0.75 \\
\hline Plasma TBARS & $1.72 \pm 0.05$ & $2.32 \pm 0.13 \dagger$ & $2.24 \pm 0.13 \dagger$ & 0.0030 \\
\hline Liver TBARS & $0.62 \pm 0.04$ & $1.05 \pm 0.09 \neq$ & $0.87 \pm 0.05^{*}$ & 0.0016 \\
\hline Kidney TBARS & $1.27 \pm 0.11$ & $1.49 \pm 0.14$ & $1.54 \pm 0.16$ & 0.37 \\
\hline Heart TBARS & $1.88 \pm 0.11$ & $2.38 \pm 0.23$ & $1.97 \pm 0.23$ & 0.20 \\
\hline Plasma CD & $31.6 \pm 2.7$ & $34.8 \pm 1.5$ & $33.0 \pm 2.0$ & 0.54 \\
\hline Liver CD & $33.7 \pm 1.0$ & $32.1 \pm 1.6$ & $29.1 \pm 2.2$ & 0.18 \\
\hline Kidney CD & $19.8 \pm 2.3$ & $20.7 \pm 1.6$ & $20.8 \pm 2.3$ & 0.93 \\
\hline Heart CD & $15.1 \pm 1.1$ & $19.5 \pm 1.4$ & $19.1 \pm 1.9$ & 0.11 \\
\hline
\end{tabular}

The parameters of oxidative stress in plasma, liver, kidney cortex and heart of SHR, SHR-Dca+/- and SHR-Dca-/- adult male rats are shown as mean \pm SEM. The significance levels of one-way ANOVA for STRAIN as a major factor are shown in the last column; significant values are italicized. ${ }^{*} p<0.05, \dagger p<0.01$ and $\neq p<$ 0.001 for pair-wise comparisons (post-hoc Tukey's HSD test) between SHR-Dca-/- or SHR-Dca+/- strains vs. SHR. ${ }^{a} p<0.05,{ }^{b} p<0.01$ and ${ }^{c} p<0.001$ for pairwise comparisons between SHR-Dca-/- and SHR-Dca+/- strains. Superoxide dismutase (SOD) units : U I ml ${ }^{-1}$ (plasma), U I mg prot ${ }^{-1}$ (liver, kidney, heart); Catalase (CAT) units: $\mu \mathrm{M} \mathrm{H}_{2} \mathrm{O}_{2} \mathrm{~min}^{-1} \mathrm{ml}^{-1}$ (plasma), $\mu \mathrm{M} \mathrm{H}_{2} \mathrm{O}_{2}$ min $^{-1} \mathrm{mg}$ prot $^{-1}$ (liver, kidney, heart); Glutathione peroxidase (GSH-Px) units $\mu \mathrm{M} \mathrm{GSH} \mathrm{min}{ }^{-1} \mathrm{ml} \mathrm{I}^{-1}$ (plasma), $\mu \mathrm{M}$ $\mathrm{GSH} \mathrm{min}^{-1} \mathrm{mg} \mathrm{prot}^{-1}$ (liver, kidney, heart); Glutathione reductase (GR) units: nM NADPH min ${ }^{-1} \mathrm{ml}^{-1}$ (plasma), nM NADPH min ${ }^{-1} \mathrm{mg} \mathrm{prot}^{-1}$ (liver, kidney, heart);

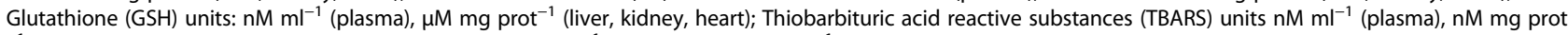

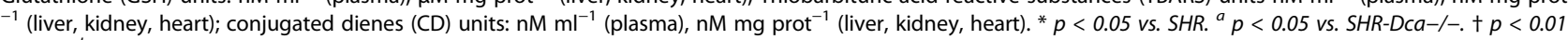
vs. SHR. ${ }^{b} p<0.01$ vs. SHR-Dca-/-. $\neq p<0.001$ vs. SHR. ${ }^{c} p<0.001$ vs. SHR-Dca-/-

oligomerize with wild type Cx50 and other connexins to form gap junction channels is well documented [23]; the ensuing functional consequences range from no effect to dominant negative inhibition of the channel function $[23,24]$. While the observable effects of the complete mutation included relative protection of the SHR-Dca -/- rats from the diabetogenic effect of dexamethasone and decrease of SOD activity combined with increase of TBARS, the heterozygous animals displayed a phenotypic profile markedly distinct from both the parental SHR and the SHR-Dca-/- strains. It has been established that gap junctions-mediated signaling is critical for correct function of pancreatic $\beta$ cells in response to glucose stimulation and pulsatile insulin release $[25$, 26]. Mice deficient in connexin36, the major connexin isoform in pancreatic $\beta$ cells, are normoglycemic but are intolerant to postprandial glucose levels and show loss of circulating insulin oscillations, similar to human prediabetes [26, 27]. A recent study of 299 type 2 diabetics and 500 unrelated normoglycemic subjects corroborated these findings in human sample by showing that $\mathrm{T}$ allele of single nucleotide polymorphism rs3743123 in GJD2 


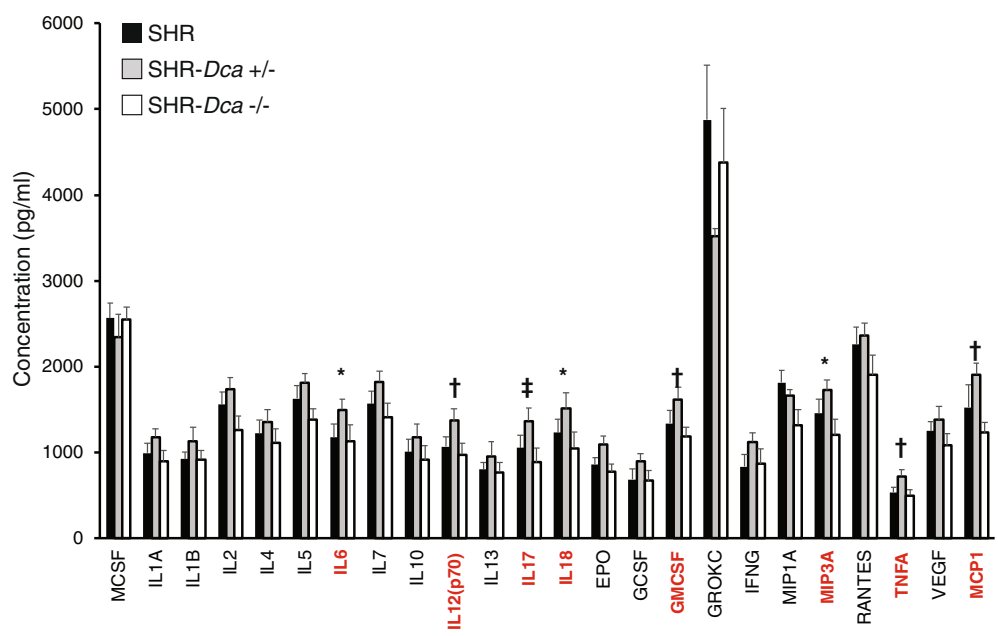

Fig. 3 The cytokine concentrations in SHR (black bars) vs. SHR-Dca+/- (grey bars) and SHR-Dca-/- (open bars) adult male rats. Data are expressed as mean \pm SEM $(n=8 /$ strain). Within the graph, the significance levels of the ANOVA for STRAIN as major factor are indicated as follows: ${ }^{*} p<0.05 ; \uparrow p<0.01 ; \neq p<0.001$. Labels of traits showing significant differences between strains are highlighted in red EPO: erythropoietin, G-CSF: granulocyte-colony stimulating factor, GM-CSF: granulocyte-macrophage colony-stimulating factor, GRO/KC: chemokine (CX-C motif) ligand 1, IFN- $\mathrm{Y}$ : interferon gamma, IL: interleukins, M-CSF: macrophage colony-stimulating factor, MCP-1: monocyte chemotactic protein 1, MIP-1a: macrophage inflammatory protein 1-alpha, MIP-3a: macrophage inflammatory protein 3-alpha, RANTES: regulated on activation, normal T cell expressed and secreted, TNF-a: tumor necrosis factor alpha, VEGF: vascular endothelial growth factor

gene (coding for Cx36) leads to altered formation of gap junction plaques and cell coupling in $\beta$ cells despite showing only marginal association to type 2 diabetes status [25]. There is further evidence showing that dexamethasone-induced insulin resistance is associated with increased connexin 36 mRNA and protein expression in pancreatic rat islets [28]. Although no data are so far available for the similar involvement of Cx50, it is of interest that according to data from the Human Integrated Protein Expression Database, CX50 protein is overexpressed in pancreas [29]. Nevertheless, this hypothesis remains to be validated by further studies. The significantly increased concentration of gastric inhibitory polypeptide together with highest insulinemia observed in heterozygotes is in line with the presumed primary action of GIP, i.e. the stimulation of glucose-dependent insulin secretion. Furthermore, the increase in postprandial insulin secretion after dexamethasone administration were shown to be mediated, at least in part, by increases in meal-stimulated GIP secretion [30]. One of the most striking observations was the simultaneous shift towards higher concentrations of triglycerides in chylomicrons and VLDL together with decrease of LDL and HDL cholesterol in heterozygous animals in comparison with both SHR and the SHR-Dca-/-. There is robust body of literature describing the role of connexins in mediating the unfavorable effects of hyperlipidemia [31-33], particularly the atherosclerotic plaque formation [34]. However, in the current study the cholesterol distribution into lipoprotein classes was modulated by distinct connexin 50 genotype, perhaps through non- genomic action of the glucocorticoid similar to the one described for Cx43 [35]. It is probable that the mutant Cx50 cannot form hemichannels or gap junctions in SHR-Dca-/- tissues [36] and their function related to lipid handling is compensated for by other members of the connexin family. However, in heterozygous animals, formation of heteromeric hemichannels containing both wild type and mutant $\mathrm{Cx} 50$ is possible with functional consequences (distinct permeability, voltage gating, ability to form homotypic or heterotypic gap junctions) leading, through yet unidentified mechanism, to the observed hyperlipidemia. Also, transdominant effects of the mutant $\mathrm{Cx} 50$ on other connexins cannot be ruled out as similar effects were described e.g. for interaction between mutant Cx26 and Cx43 [37]. Several of the increased oxidative stress indices were apparent in plasma and livers of rats with both homozygous and heterozygous form of the Cx50 mutation. Again, this trend was much more pronounced in SHR-Dca+/- rats, with exclusive manifestation also in kidney (decrease of activity glutathione peroxidase and superoxide dismutase). This was accompanied by increased levels of proinflammatory cytokines including IL-6, IL-12, IL-17 and TNF- $\alpha$. The role of oxidative stress and inflammation in cardiovascular disease and related conditions is well established and is affected by numerous intrinsic and extrinsic factors [38]. Several connexins were previously linked to redox homeostasis, including Cx40 [39], Cx43 [40] and Cx46 [41]. The mechanism of their involvement include intra- and extracellular exchange of small molecules that are critical for redox homeostasis, such as 
GSH [42], for which Cx50 was shown to be permeable [41]. The heteromeric channels containing the mutant Cx50 could thus affect the redox homeostasis directly by modulating the flow of relevant ions, molecules or metabolites or through altered post-translational modifications and interactions with other cellular proteins [43]. One of the limitations of the present study lies in the fact that we did not uncover the detailed mechanism which underlies the metabolic effects in the Cx50-deficient and heterozygous animals. Also, since all the measurements were done in animals treated with dexamethasone, the specific outcomes may be due to pharmacogenetic interaction of variant Cx50 with dexamethasone as glucocorticoid effects on connexins have been documented [35].

\section{Conclusions}

The results of this study demonstrate that homozygous mutation in the amino terminal domain of Cx50 leads, in dexamethasone-treated adult male rats, to relative protection from dexamethasone-induced insulin resistance, decrease in visceral adiposity and increase in oxidative stress indices. At the same time, heterozygous form of the same Cx50 variant results in increased concentration of triacylglycerols, decrease of cholesterol and elevation of several pro-inflammatory cytokines. Altogether, we show the substantial involvement of connexin 50 in several metabolic syndrome features.

\section{Additional file}

Additional file 1: Table S1. Lipoprotein particle size in SHR, SHR-Dca+/and SHR-Dca-/- male rats. (PDF $114 \mathrm{~kb}$ )

\footnotetext{
Abbreviations

C: Cholesterol; CAT: Catalase; CD: Conjugated dienes; CM: Chylomicron; Cx26: Connexin26; Cx36: Connexin36; Cx40: Connexin40; Cx43: Connexin43; Cx46: Connexin46; Cx50: Connexin50; EPF: Epididymal fat pad;

EPO: Erythropoietin; G-CSF: Granulocyte-colony stimulating factor; GIP: Gastric inhibitory peptide; Gja8 gene: Gap junction protein, alpha 8 coding for connexin50 gene; GJD2 gene: Gap junction protein, delta 2 coding for connexin36 gene; GLP1: Glucagon-like polypeptide-1; GM-CSF: Granulocytemacrophage colony-stimulating factor; GR: Glutathione reductase; GRO/ KC: Chemokine (C-X-C motif) ligand 1; GSH: Glutathione; GSH-PX: Glutathione peroxidase; HDL: High-density lipoprotein; IFN-y: Interferon gamma; IL10: Interleukin 10; IL-12(p70): Interleukin 12(p70); IL-13: Interleukin 13; IL17A: Interleukin 17A; IL-18: Interleukin 18; IL-1a: Interleukin 1a; IL1ß: Interleukin 13; IL-2: Interleukin 2; IL-4: Interleukin 4; IL-5: Interleukin 5; IL6: Interleukin 6; IL-7: Interleukin 7; LDL: Low-density lipoprotein; MCP-

1: Monocyte chemotactic protein 1; M-CSF: Macrophage colony-stimulating factor; MIP-1a: Macrophage inflammatory protein 1-alpha; MIP-

3a: Macrophage inflammatory protein 3-alpha; OGTT: Oral glucose tolerance test; PP: Pancreatic polypeptide; PYY: Protein tyrosine tyrosine; RANTES: Regulated on activation, normal T cell expressed and secreted; RPF: Retroperitoneal fat pad; SHR: Spontaneously hypertensive rat; SHR-Dca -/-: Coisogenic SHR strain with a spontaneous mutation (homozygous) L7Q in Gja8 gene, SHR-Gja $8^{\text {m1Cub-/-; }}$; SHR-Dca+/-: Coisogenic SHR strain with a spontaneous mutation (heterozygous) L7Q in Gja8 gene, SHR-Gja8 ${ }^{\text {miCubt/- }}$; SOD: Superoxide dismutase; TBARS: Thiobarbituric acid reactive substances;
}

TG: Triacylglycerols; TNF-a: Tumor necrosis factor alpha; VEGF: Vascular endothelial growth factor; VLDL: Very low-density lipoprotein

\section{Acknowledgements}

Not applicable.

\section{Funding}

This work was supported by Czech Science Foundation Project GAČR P301/ 12/0777, Project LK11217 from the Ministry of Education, Youth and Sports of the Czech Republic and Charles University in Prague [PRVOUK-P25/LF1/2, UNCE 204022].

\section{Availability of data and materials}

The data that support the findings of this study are available from the corresponding author upon reasonable request.

\section{Authors' contributions}

VK and DK derived the SHR-Dca+/- and SHR-Dca-/- strains. OS, LS, MK and $\mathrm{BC}$ carried out the metabolic component of the study and drafted the manuscript. FL participated in the design of the study and performed the statistical analysis. LK and $\mathrm{OO}$ performed the assessment of oxidative stress indices. OS, VK and LK conceived the study, and participated in its design and coordination and helped to draft the manuscript. All authors participated in the manuscript preparation, read and approved the final manuscript.

\section{Competing interests}

The authors declare that they have no competing interests.

\section{Consent for publication}

Not applicable.

\section{Ethics approval}

This project was performed in conformity with the Animal Protection Law of the Czech Republic. The experimental protocols and detailed procedures were evaluated and approved by the Ethical Committee of the First Faculty of Medicine, Charles University in Prague and by the Ministry of Education, Youth and Sports of the Czech Republic. The health of the rats was examined daily, and the animals were monitored every hour during the experimental procedures. There were no unexpected deaths during the experiment. Overdose of anesthetic (halothane) was the method of euthanasia in this study. All efforts were made to minimize suffering of the experimental animals.

\section{Author details}

'Institute of Biology and Medical Genetics, First Faculty of Medicine, Charles University, Albertov 4, 12800 Praque 2, Czech Republic. ${ }^{2}$ Division BIOCEV, Institute of Molecular Genetics of the Academy of Sciences of the Czech Republic, Laboratory of Rat Models of Metabolic Disorders, Vídeňská 1083, 14220 Prague 4, Czech Republic. ${ }^{3}$ Center for Experimental Medicine, Institute for Clinical and Experimental Medicine, Vídeňská 1958/9, 14021 Prague 4, Czech Republic.

Received: 25 May 2016 Accepted: 14 November 2016 Published online: 21 November 2016

\section{References}

1. Alberti KG, Eckel RH, Grundy SM, Zimmet PZ, Cleeman Jl, Donato KA, Fruchart JC, James WP, Loria CM, Smith Jr SC, et al. Harmonizing the metabolic syndrome: a joint interim statement of the International Diabetes Federation Task Force on Epidemiology and Prevention; National Heart, Lung, and Blood Institute; American Heart Association; World Heart Federation; International Atherosclerosis Society; and International Association for the Study of Obesity. Circulation. 2009;120:1640-5.

2. Lusis AJ, Attie AD, Reue K. Metabolic syndrome: from epidemiology to systems biology. Nat Rev Genet. 2008:9:819-30.

3. Seda O, Tremblay J, Sedova L, Hamet P. Integrating genomics and transcriptomics with geo-ethnicity and the environment for the resolution of complex cardiovascular diseases. Curr Opin Mol Ther. 2005;7:583-7.

4. Ciccone MM, Scicchitano P, Cameli M, Cecere A, Cortese F, et al. Endothelial Function in Pre-diabetes, Diabetes and Diabetic Cardiomyopathy: A Review. J Diabetes Metab. 2014;5:364. doi:10.4172/2155-6156.1000364. http://www. 
omicsonline.org/open-access/endothelial-function-in-prediabetes-diabetesand-diabetic-cardiomyopathy-2155-6156.1000364.php?aid=25586.

5. Aitman TJ, Boone C, Churchill GA, Hengartner MO, Mackay TF, Stemple DL. The future of model organisms in human disease research. Nat Rev Genet. 2011;12:575-82.

6. Saez JC, Leybaert L. Hunting for connexin hemichannels. FEBS Lett. 2014; 588:1205-11.

7. Hamelin R, Allagnat F, Haefliger JA, Meda P. Connexins, diabetes and the metabolic syndrome. Curr Protein Pept Sci. 2009;10:18-29.

8. Shimoyama M, De Pons J, Hayman GT, Laulederkind SJ, Liu W, Nigam R, Petri V, Smith JR, Tutaj M, Wang SJ, et al. The Rat Genome Database 2015: genomic, phenotypic and environmental variations and disease. Nucleic Acids Res. 2015:43:D743-50.

9. Liska F, Chylikova B, Martinek J, Kren V. Microphthalmia and cataract in rats with a novel point mutation in connexin 50-L7Q. Mol Vis. 2008;14:823-8.

10. Krupkova M, Liska F, Pravenec M, Zidek V, Vernerova Z, Krenova D, Kren V, Seda O. Connexin50 mutation L7Q attenuates hypertension in spontaneously hypertensive rat SHR/Olalpcv. Eur J Hum Genet. 2013;21 Suppl 2:247.

11. Krupkova M, Sedova L, Liska F, Krenova D, Kren V, Seda O. Pharmacogenetic interaction between dexamethasone and $\mathrm{Cd} 36$-deficient segment of spontaneously hypertensive rat chromosome 4 affects triacylglycerol and cholesterol distribution into lipoprotein fractions. Lipids Health Dis. 2010;9:38.

12. Seda O, Liska F, Krenova D, Kazdova L, Sedova L, Zima T, Peng J, Pelinkova K, Tremblay J, Hamet P, Kren V. Dynamic genetic architecture of metabolic syndrome attributes in the rat. Physiol Genomics. 2005;21:243-52.

13. Sedova L, Liska F, Krenova D, Kazdova L, Tremblay J, Krupkova M, Corbeil G, Hamet P, Kren V, Seda O. CD36-deficient congenic strains show improved glucose tolerance and distinct shifts in metabolic and transcriptomic profiles. Heredity (Edinb). 2012;109:63-70.

14. Usui S, Hara Y, Hosaki S, Okazaki M. A new on-line dual enzymatic method for simultaneous quantification of cholesterol and triglycerides in lipoproteins by HPLC. J Lipid Res. 2002;43:805-14.

15. Seda O, Sedova L, Oliyarnyk O, Kazdova L, Krenova D, Corbeil G, Hamet P, Tremblay J, Kren V. Pharmacogenomics of metabolic effects of rosiglitazone. Pharmacogenomics. 2008;9:141-55.

16. Malinska H, Oliyarnyk O, Hubova M, Zidek V, Landa V, Simakova M, Mlejnek P, Kazdova L, Kurtz TW, Pravenec M. Increased liver oxidative stress and altered PUFA metabolism precede development of non-alcoholic steatohepatitis in SREBP-1a transgenic spontaneously hypertensive rats with genetic predisposition to hepatic steatosis. Mol Cell Biochem. 2010:335:119-25.

17. Naito C, Kawamura M, Yamamoto Y. Lipid peroxides as the initiating factor of atherosclerosis. Ann N Y Acad Sci. 1993;676:27-45.

18. Lubkemeier I, Machura K, Kurtz L, Neubauer B, Dobrowolski R, Schweda F, Wagner C, Willecke K, Kurtz A. The connexin 40 A96S mutation causes renin-dependent hypertension. J Am Soc Nephrol. 2011;22:1031-40.

19. Kurtz A. Connexins, renin cell displacement and hypertension. Curr Opin Pharmacol. 2015;21:1-6.

20. Beyer EC, Ebihara L, Berthoud VM. Connexin mutants and cataracts. Front Pharmacol. 2013;4:43.

21. Xin $L$, Bai D. Functional roles of the amino terminal domain in determining biophysical properties of Cx50 gap junction channels. Front Physiol. 2013;4:373.

22. Xin L, Sun Y, Bai D. Heterotypic connexin50/connexin50 mutant gap junction channels reveal interactions between two hemichannels during transjunctional voltage-dependent gating. J Physiol. 2012;590:5037-52.

23. Tong JJ, Minogue PJ, Guo W, Chen TL, Beyer EC, Berthoud VM, Ebihara L. Different consequences of cataract-associated mutations at adjacent positions in the first extracellular boundary of connexin50. Am J Physiol Cell Physiol. 2011;300:C1055-64.

24. Pal JD, Berthoud VM, Beyer EC, Mackay D, Shiels A, Ebihara L. Molecular mechanism underlying a Cx50-linked congenital cataract. Am J Physiol. 1999;276:C1443-6

25. Cigliola V, Populaire C, Pierri CL, Deutsch S, Haefliger JA, Fadista J, Lyssenko V, Groop L, Rueedi R, Thorel F, et al. A Variant of GJD2, Encoding for Connexin 36, Alters the Function of Insulin Producing beta-Cells. PLoS One. 2016;11:e0150880.

26. Head WS, Orseth ML, Nunemaker CS, Satin LS, Piston DW, Benninger RK. Connexin-36 gap junctions regulate in vivo first- and second-phase insulin secretion dynamics and glucose tolerance in the conscious mouse. Diabetes. 2012:61:1700-7.
27. Meda P. The in vivo beta-to-beta-cell chat room: connexin connections matter. Diabetes. 2012:61:1656-8.

28. Rafacho A, Roma LP, Taboga SR, Boschero AC, Bosqueiro JR. Dexamethasone-induced insulin resistance is associated with increased connexin 36 mRNA and protein expression in pancreatic rat islets. Can J Physiol Pharmacol. 2007;85:536-45.

29. Fishilevich S, Zimmerman S, Kohn A, Iny Stein T, Olender T, Kolker E, Safran $\mathrm{M}$, Lancet $\mathrm{D}$. Genic insights from integrated human proteomics in GeneCards Database. 2016. 2016:baw030. doi:10.1093/database/baw030 published online April 5, 2016. http://database.oxfordjournals.org/content/ 2016/baw030.

30. Cummings BP, Bremer AA, Kieffer TJ, D'Alessio D, Havel PJ. Investigation of the mechanisms contributing to the compensatory increase in insulin secretion during dexamethasone-induced insulin resistance in rhesus macaques. J Endocrinol. 2013;216:207-15.

31. Gorbe A, Varga ZV, Kupai K, Bencsik P, Kocsis GF, Csont T, Boengler K, Schulz $R$, Ferdinandy P. Cholesterol diet leads to attenuation of ischemic preconditioning-induced cardiac protection: the role of connexin 43. Am J Physiol Heart Circ Physiol. 2011;300:H1907-13.

32. Li T, Yang GM, Zhu Y, Wu Y, Chen XY, Lan D, Tian KL, Liu LM. Diabetes and hyperlipidemia induce dysfunction of VSMCs: contribution of the metabolic inflammation/miRNA pathway. Am J Physiol Endocrinol Metab. 2015;308:E257-69

33. Haefliger JA, Martin D, Favre D, Petremand $Y$, Mazzolai L, Abderrahmani A, Meda P, Waeber G, Allagnat F. Reduction of connexin36 content by ICER-1 contributes to insulin-secreting cells apoptosis induced by oxidized LDL particles. PLoS One. 2013;8:e55198.

34. Pfenniger A, Chanson M, Kwak BR. Connexins in atherosclerosis. Biochim Biophys Acta. 1828;2013:157-66.

35. Samarasinghe RA, Di Maio R, Volonte D, Galbiati F, Lewis M, Romero G, DeFranco DB. Nongenomic glucocorticoid receptor action regulates gap junction intercellular communication and neural progenitor cell proliferation. Proc Natl Acad Sci U S A. 2011;108:16657-62.

36. Rubinos C, Villone K, Mhaske PV, White TW, Srinivas M. Functional effects of Cx50 mutations associated with congenital cataracts. Am J Physiol Cell Physiol. 2014;306:C212-20.

37. Shuja Z, Li L, Gupta S, Mese G, White TW. Connexin26 Mutations Causing Palmoplantar Keratoderma and Deafness Interact with Connexin43, Modifying Gap Junction and Hemichannel Properties. J Invest Dermatol. 2016;136:225-35.

38. Ciccone MM, Cortese F, Gesualdo M, Carbonara S, Zito A, Ricci G, De Pascalis F, Scicchitano P, Riccioni G. Dietary intake of carotenoids and their antioxidant and anti-inflammatory effects in cardiovascular care. Mediators Inflamm. 2013:2013:782137.

39. Yu G, Bolon M, Laird DW, Tyml K. Hypoxia and reoxygenation-induced oxidant production increase in microvascular endothelial cells depends on connexin40. Free Radic Biol Med. 2010;49:1008-13.

40. Le HT, Sin WC, Lozinsky S, Bechberger J, Vega JL, Guo XQ, Saez JC, Naus CC. Gap junction intercellular communication mediated by connexin 43 in astrocytes is essential for their resistance to oxidative stress. J Biol Chem. 2014;289:1345-54.

41. Slavi N, Rubinos C, Li L, Sellitto C, White TW, Mathias R, Srinivas M. Connexin 46 (cx46) gap junctions provide a pathway for the delivery of glutathione to the lens nucleus. J Biol Chem. 2014;289:32694-702.

42. Gao K, Chi Y, Zhang X, Zhang H, Li G, Sun W, Takeda M, Yao J. A novel TXNIP-based mechanism for Cx43-mediated regulation of oxidative drug injury. J Cell Mol Med. 2015;19:2469-80.

43. Pogoda K, Kameritsch P, Retamal MA, Vega JL. Regulation of gap junction channels and hemichannels by phosphorylation and redox changes: a revision. BMC Cell Biol. 2016;17 Suppl 1:11. 